\title{
The in vitro and in vivo treatment effects of overexpressed lentiviral vector-mediated human BMP2 gene in the femoral bone marrow stromal cells of osteoporotic rats
}

\author{
BING ZHAO ${ }^{1}$, QIANG WANG ${ }^{1}$, TIANZUN TAO ${ }^{1}$, JUAN LI $^{2}$ and QINGYUAN LIN ${ }^{3,4}$ \\ Departments of ${ }^{1}$ Orthopedics and ${ }^{2}$ Clinical Laboratory Medicine, the Second Hospital Affiliated to \\ Harbin Medical University; ${ }^{3}$ Department of Immunology, Harbin Medical University; ${ }^{4}$ Immunity \\ and Infection Key Laboratory of Heilongjiang Province, Harbin 150081, P.R. China
}

Received July 4, 2013; Accepted September 2, 2013

DOI: $10.3892 /$ ijmm.2013.1507

\begin{abstract}
This study aimed to compare the treatment effects of lentiviral vector-mediated hBMP2 which was overexpressed in the femoral bone marrow stromal cells of osteoporotic rats through genetic infection in vitro and in vivo. Comparison of the two transgenic effects may be crucial to determining the lentivirus infection method to be used. Following a comparison of the rat bone marrow stromal cells (rBMSCs) in osteoporotic (MSCs OVX) and normal (MSCs $\mathrm{CON}$ ) groups, the lentiviral vector-mediated human bone morphogenetic protein 2 (hBMP2), which overexpressed the BMSCs of osteoporotic rats in vitro (rBMSCs in OE group), was constructed. The osteogenic ability in the overexpressed (OE) group was then compared to that of the MSCs CON. The rBMSCs in the OE group (transplants of genetic infection in vitro) and the lentivirus-containing solution (injected material of genetic infection in vivo) were injected into the femurs. The treatment effect of each group was compared via bone mineral density (BMD) and bone histomorphometry. The hBMP2-modified osteoporosis rBMSCs formed by genetic infection in vitro $(\mathrm{n}=7)$ had an ameliorated treatment effect on the femur as compared to that of the in vivo $(\mathrm{n}=7)$ (BMD: 0.315 vs. $0.19 \mathrm{~g} / \mathrm{cm}^{2}, \mathrm{P}<0.01$; bone histomorphometry: For bone trabeculars (Tb.Ar/T.Ar): 0.301 vs. $0.114, \mathrm{P}<0.01$; for trabecular thickness (Tb.Th): 43.54 vs. $21.39 \mu \mathrm{m}, \mathrm{P}<0.01$; for trabecular separation (Tb.Sp): 115.7 vs. $304.87 \mu \mathrm{m}, \mathrm{P}<0.01$ ). The results showed that the treatment effects of osteoporotic rBMSCs on local osteoporosis performed by genetic infection were improved in vitro as compared to those in vivo.
\end{abstract}

Correspondence to: Dr Tianzun Tao, Department of Orthopedics, the Second Hospital Affiliated to Harbin Medical University, No. 148 Baojian Road, Harbin 150081, P.R. China

E-mail: taotianzunttz@yahoo.com

Key words: local osteoporosis, bone morphogenetic protein 2, bone marrow stromal cells, genetic infection in vitro, genetic infection in vivo

\section{Introduction}

Human bone morphogenetic protein 2 (hBMP2) is a protein of ossification differentiation, is secreted by human bone marrow stromal cells (BMSCs), and is an early regulatory substance $(1,2)$. The ossification differentiation of BMSCs is a process that involves various types of BMPs that adjust to each other in a manner similar to that of a reticular system $(3,4)$. The BMP2 improves its own output as well as that of other endogenous BMPs secreted from BMSCs (5). Other BMPs have shown an increase with an increase in the endogenous BMP2, while no marked difference exists in the ability of the body to respond to BMP2 at different ages (6). Therefore, BMP2 was used as the target gene for the treatment of local osteoporosis in rat femurs. Recent studies have confirmed that BMP2 is capable of promoting ossification $(1,2,7,8)$. However, a number of limiting factors, including the difficulty of isolating natural BMP, tolerating the temperature at $37^{\circ} \mathrm{C}$ in in vivo environments and maintaining effective density as well as short half-life, have made the application of BMP difficult (9).

If the target gene were imported, coding the cell factor protein into appropriate target cells through transgenic techniques, the target cells would continuously secrete this protein in order to act on the target cells in an autocrine or paracrine manner. Therefore, by utilizing this process, the problem of local application of exogenous cell factor would likely be resolved (10-12). In this study, the density of hBMP2 in marrow cavity was adjusted according to the average volume of the grown Sprague-Dawley rat femoral marrow cavity as well as the expression level of hBMP2 secreted by hBMP2 gene-modified BMSCs of osteoporotic rats. The density of hBMP2 proteins in the rat femoral marrow cavity was set to $0.15 \mathrm{mg} / \mathrm{ml}$, making the BMSCs osteogenic and enabling the treatment of local osteoporosis without any side effects, such as cyst-like bone formation or soft tissue swelling $(13,14)$.

Lentivirus is a common transgenic carrier used in laboratories due to its higher infection efficiency and more stable expression of transfected genes $(10,12-15)$. There are two methodologies for lentivirus transfection, genetic transfection in vivo and in vitro $(16,17)$. The definition of genetic transfection in vivo is the injection of the lentivirus carrying the 
target gene into the relevant body part according to a certain multiplicity of infection (MOI), which could have a transgenic effect on stem cells. The definition of genetic infection in vitro is the isolation of the stem cells first and then mixing of the lentivirus with these cells according to a certain MOI, followed by transplantation of the infected cells into the relevant body part. The two transgenic effects caused by these two procedures have been previously demonstrated (18-21). Additionally, a comparison of the two transgenic effects may be crucial to determining the lentivirus infection method to be used.

In most in vitro or in vivo genetic infection experiments with lentivirus, normal BMSCs were used as the experimental materials (20-23). However, if normal human BMSCs were to be used in clinical practice, osteoporosis patients could face many practical issues, including shortage of normal BMSCs, excessive treatment costs and ethical/moral questions. Since the twentieth century, medical technology has been radically transformed as a modern science with a focus on numerous technological breakthroughs. However, the accessibility of such technology, its cost-effectiveness and the adverse effects to the society remain to be clarified. Subsequently, the BMSCs of osteoporotic rats were used as the experimental materials to treat local osteoporosis in rat femurs. The long-term purpose of our experiment was to lay the foundation for the treatment of local osteoporosis in patients by transplanting their own BMSCs infected with overexpressed genes that could ameliorate local osteoporosis. Additionally, the aim was to identify an economically viable treatment for local osteoporosis.

\section{Materials and methods}

Study design. Sprague-Dawley rats (SD rats) were divided into the: osteoporosis, normal and sham groups. Postmenopausal osteoporosis models were constructed by removing the ovaries in the osteoporotic group. The control group was created by removing the adipose tissue around the ovaries in the sham group. After three months, the rat BMSCs (rBMSCs) were isolated from the femurs in the osteoporotic and normal groups and were compared by colony-forming units-fibroblastic (CFU-F) counting, osteoblast ALP staining (improved Gomori calcium-cobalt method), and osteogenic capability analysis (ALP activity, OCN level). The average number of rBMSCs isolated from a femur in each group was calculated based on the CFU-F counting. The osteogenic capability of rBMSCs was compared among groups by osteoblast ALP staining and osteogenic capability analysis. The lentiviral vector-mediated hBMP2 overexpressed in rBMSCs was constructed to be used in the osteoporotic group in vitro (rBMSCs in OE group). The osteogenic capability (ALP activity, OCN level) of rBMSCs in the OE group was compared to that of the MSCs CON lengthways. After the comparison, five study groups were designed using SD rats, including the transplanted group of lentiviral vector-mediated hBMP2 overexpressed (OE) cells, transplanted group of lentiviral vector-mediated hBMP2 negative control (NC) cells, injected group of lentivirus, transplanted group of rat bone marrow stromal (MSCs OVX) cells and the saline control group. Postmenopausal osteoporosis models were constructed again with all five groups. The rBMSCs in the OE group (transplants of genetic infection in vitro), lentivirus-containing solution (injected material of genetic infection in vivo) and some control materials were injected into the osteoporotic femurs of the corresponding groups. After three months, the treatment effects were compared in each group through whole femoral BMD and bone histomorphometry in the distal part of the femur.

Animals and osteoporosis models. Forty-one female SD rats of $\sim 6$-month-old, weighing 400-430 g at the start of the experiment, were obtained from the Amimal Experiment Center of the Second Affiliated Hospital, Harbin Medical University. Rats were randomly divided into the osteoporosis (MSCs OVX), normal (MSCs CON) and sham (SHAM CON) groups. The MSCs OVX and MSCs CON groups comprised 17 rats each and the SHAM CON comprised 7 rats. The rats in the MSCs OVX and SHAM CON groups were anesthetized by intraperitoneal injection of $5 \%$ chloralic hydras $(0.66 \mathrm{ml} / 100 \mathrm{~g})$. The ovaries of rats in MSCs OVX were removed to construct the postmenopausal osteoporosis models (PO models) and the adipose tissue around the ovaries of rats in SHAM CON were removed in order to create a control group. After three months, 7 rats were sacrificed from each group. Measurements were obtained for: content of estrogens in serum, bone mineral density (BMD) of the right femoral distal 1/3 section, uterus wet weight and left femur dry weight. Furthermore, sections of the right femur were stained to verify the PO models. Subsequent to verification of the PO models, 10 rats were left in the MSCs OVX and MSCs CON groups, respectively, while no rats were left in the SHAM CON group. The use of the samples was approved by the local healthcare authorities and Ethics Committee (The Guide for the Care and Use of Laboratory Animals published by the US National Institutes of Health, NIH Publication No. 85-23, revised 1985).

Isolation of rBMSCs. The nucleated cells containing rBMSCs were isolated from the left femurs of the remaining SD rats in the MSCs OVX and MSCs CON groups under sterile conditions according to the protocol reported by Maniatopoulos et al (24). Briefly, the two ends of the femora were cut off at the epiphysis and marrow was flushed out using low glucose Dulbecco's modified Eagle's medium (L-DMEM) (Gibco BRL, Grand Island, NY, USA) supplemented with $10 \%$ (v/v) fetal bovine serum (FBS) (HyClone, Logan, UT, USA) and $200 \mathrm{U} / \mathrm{ml}$ of heparin (Sigma, St. Louis, MO, USA). The cells were gently kneaded in L-DMEM containing $10 \%$ FBS in order to obtain a single-cell suspension. Trypan blue (Guangzhou Genebase Bioscience Co., Ltd., Guangzhou, China) staining was used to analyze the cell activity prior to cell culture. The percentage of cell activity was calculated according to the formula: Cell activity $(\%)=[($ total cells - colored cells $) /$ total cells $] \times 100 \%$. The percentage of cell activity was $>96 \%$ in all the groups. Procedures were performed at a facility accredited by the Institutional Animal Care and Use Committee of Harbin Medical University. The culture medium was changed on the 6th day for the first time and then non-adherent cells were abandoned. When $90 \%$ confluency was reached, the BMSCs were released from the culture substratum using trypsin/EDTA $(0.25 \% \mathrm{w} / \mathrm{v}$ trypsin, $0.02 \%$ EDTA) and were moved to culture dishes $\left(10 \mathrm{~cm}\right.$ in diameter) at $1.0 \times 10^{5}$ cells $/ \mathrm{ml}$ in $10 \mathrm{ml}$. The characterization of rat BMSCs was validated by a flow cytometry assay, as previously described (25). 
Counting of $C F U-F s$. Passage (P) 0 was randomly selected from each group in rBMSCs $(n=5)$. The method of CFU-Fs counting was performed according to the protocol reported by Nishida et al (26). The cells isolated from the rat femur were gently kneaded and $10 \mathrm{ml}$ of single-cell suspension was prepared with all the cells (suspension A). We drew $10 \mu \mathrm{l}$ of suspension A (suspension B) and dispersed it in $10 \mathrm{ml}$ of L-DMEM containing $10 \%$ FBS to prepare suspension C. A total of $2.5 \mathrm{ml}$ of suspension $\mathrm{C}$ was cultured in each well of a 6 -well plate. The media were changed on the sixth day for the first time and the non-adherent cells were abandoned. On the 10th day, the plate was placed under an inverted microscope and the number of CFU-F was counted in every well.

ALP staining of osteoblasts. The improved Gomori calciumcobalt method (27) was utilized to perform ALP staining in osteoblasts. P2 rBMSCs were chosen in each group $(n=3)$ to produce rBMSCs suspension in osteogenic induction medium (OI medium) containing high-glucose Dulbecco's modified Eagle's medium (H-DMEM) (Gibco BRL) including $0.1 \mu \mathrm{mol} / 1$ dexamethasone, $10 \mathrm{mmol} / 1 \beta$-glycerophosphate, and $50 \mathrm{mg} / \mathrm{l}$ ascorbic acid. The rBMSCs were cultured at a density of $1 \times 10^{5} / \mathrm{ml}$. The OI medium was changed every three days. After 28 days, the cells were removed and cultured in 24-well plates containing sterile cell slides (Nunc, Denmark) at a density of $2 \times 10^{4} / \mathrm{ml}$ with the same OI medium for five days. The cell slides were prepared with $95 \%$ ethanol, incubation solution $(5 \mathrm{ml}$ of $3 \% \beta$-glycerophosphate; $5 \mathrm{ml}$ of $2 \%$ barbital sodium; $10 \mathrm{ml}$ of distilled water; $10 \mathrm{ml}$ of $2 \% \mathrm{CaCl}_{2}$; and $1 \mathrm{ml}$ of $2 \% \mathrm{MgSO}_{4}$ ), $2 \%$ cobalt nitrate, and $1 \%$ ammonium sulphate. Images of the stained rBMSCs after OI were captured in each group under a confocal laser scanning microscope (FV1000, Olympus, Tokyo, Japan).

Osteogenic capability analysis. ALP activity and the osteocalcin (OCN) level were detected, which reflected the osteogenic ability of rBMSCs. P3 rBMSCs in each group $(n=5)$ were chosen to produce the rBMSC suspensions at a density of $1 \times 10^{5} / \mathrm{ml}$ by OI media. The OI media were changed every three days. The rBMSC ALP activity was separately detected on the 7th and 14th day of OI. The rBMSC supernatants were separately collected on the 14th, 21st, and 28th day in order to detect the OCN level. The ALP activity assay kit (Sigma) using p-nitrophenyl phosphate (p-NPP) as a substrate in order was employed to determine the rBMSC ALP activity. Rat OCN enzyme-linked immunoabsorbent assay (ELISA) kit (Westang Bio-tech, Shanghai, China) was used to detect the OCN level.

Lentiviral vector construction, virus production and infection. The hBMP2 cDNA was amplified from the human osteosarcoma MG-63 cell line by polymerase chain reaction (PCR) with the primers described in Table I. The hBMP2 cDNA was subcloned in the lentiviral vector-plasmid (GV208, Ubi-MCSEGFP, Genechem, Shanghai, China), and the hBMP2 gene overexpressing the lentiviral vector-plasmid (Ubi-MCShBMP2-EGFP) was then utilized.

Lentiviral vector-plasmid (Ubi-MCS-EGFP) and hBMP2 gene overexpressing lentiviral vector-plasmid (Ubi-MCShBMP2-EGFP) were used to transfect the $293 \mathrm{~T}$ cells using Lipofectamine $^{\mathrm{TM}} 2000$ (Invitrogen Life Technologies, Carlsbad,
Table I. hBMP2 primers. ${ }^{a}$

\begin{tabular}{lc}
\hline Gene & Primers \\
\hline hBMP2 & 5'-GAGGATCCCCGGGTACCGGTCGCCACCA \\
& TGGTGGCCGGGACCCGCTGTC-3' (forward) \\
& 5'-TCACCATGGTGGCGACCGGGCGACACCC \\
& ACAACCCTCCAC-3' (reverse)
\end{tabular}

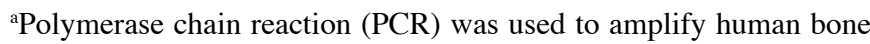
morphogenetic protein 2 (hBMP2) gene from the human osteosarcoma MG-63 cell line

CA, USA). Therefore, the lentivirus containing hBMP2 cDNA (Lv-BMP) as well as that containing GFP cDNA (Lv-GFP) were obtained for subsequent analysis. The lentivirus titer was detected by quantitative PCR (qPCR) (the titer of Lv-GFP: $8 \mathrm{E}+8 \mathrm{TU} / \mathrm{ml}$, the titer of Lv-BMP: $2 \mathrm{E}+8 \mathrm{TU} / \mathrm{ml}$ ).

At MOI of 20 and 40 , in the presence of $8 \mu \mathrm{g} / \mathrm{ml}$ polybrene (Sigma), P3 rBMSCs of MSCs OVX were infected with Lv-GFP and designated as the hBMP2-negative control group (NC group). Similarly, the P3 rBMSCs of MSCs OVX were infected with Lv-BMP and were designated as the hBMP2 OE group (Fig. 1). According to the MOI gradient, five groups ( $n=3$ per group) were identified: rBMSCs of MSCs OVX not infected with lentivirus (control group), rBMSCs of MSCs OVX infected with Lv-BMP at a MOI of 20 (low OE group), rBMSCs of MSCs OVX infected with Lv-BMP at a MOI of 40 (high OE group), rBMSCs of MSCs OVX infected with Lv-GFP at a MOI of 20 (low NC group) and rBMSCs of MSCs OVX infected with Lv-GFP at a MOI of 40 (high NC group). The aim was to identify a better MOI capable of producing better infection by detecting and comparing the relative mRNA expression in these groups.

qPCR analysis. Gene-specific primer sets were designed for hBMP2 and glyceraldehyde-3-phosphate dehydrogenase (GAPDH) (Table II) to detect the relative mRNA expression. The rBMSCs were collected from all five groups described above on day seven after the infection. Total RNA was extracted using the TRIzol (Invitrogen Life Technologies) from each cell sample and the cDNA was synthesized from the total RNA. qPCR was carried out using SYBR-Green I (Molecular Probes, Eugene, OR, USA) in a Rotor-Gene 3000 (Corbett Research, Sydney, Australia). To correct for differences in the RNA quality and quantity among samples, the data were normalized to those of the GAPDH. The melting curves were made after the qPCR was finished.

ELISA analysis. According to the improved MOI detected by qPCR analysis, the P3 rBMSCs in MSCs OVX were separately infected by Lv-BMP and Lv-GFP. The infected rBMSCs were separately designated as P3' rBMSCs in the OE and $\mathrm{NC}$ groups. The rBMSCs lost contact inhibition, i.e., the cell growth rate was retarded and the cells began to overlap and gather on the 9th day as verified through direct observation of the P3' rBMSCs in the OE group with OI media. While the BMSCs lost contact inhibition, the cells began to form 

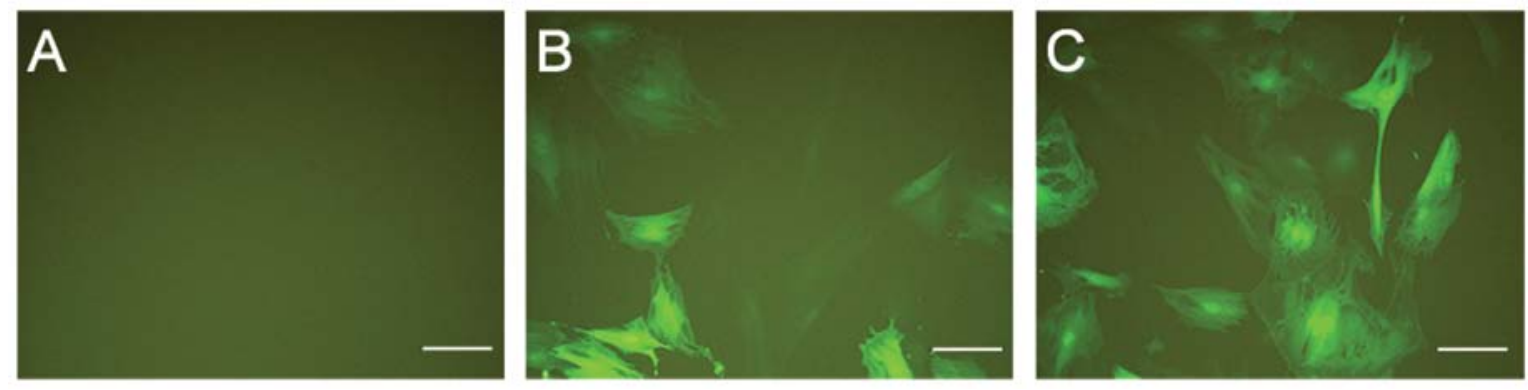

Figure 1. (A) Rat bone marrow stromal cells (rBMSCs) of osteoporotic (MSCs OVX) cells not infected with lentivirus (control group). (B) The rBMSCs of MSCs OVX infected with the lentivirus containing hBMP2 cDNA (Lv-BMP) at a multiplicity of infection (MOI) of 20 (low OE group). (C) The rBMSCs of MSCs OVX infected with Lv-BMP at a MOI of 40 (high OE group). An infection efficiency of up to $80 \%$ was obtained in the low OE and high OE groups, separately determined by the fraction of fluorescent cells under fluorescent microscopy at three days after infection (Bar, $125 \mu \mathrm{m})$.

Table II. Gene-specific primers for hBMP2 and GAPDH. ${ }^{a}$

\begin{tabular}{ll}
\hline Gene & \multicolumn{1}{c}{ Primers } \\
\hline hBMP2 & 5'-CATGCCATTGTTCAGACG-3' (forward) \\
& 5'-TGTACTAGCGACACCCACA-3' (reverse) \\
GAPDH & 5'-TTCAACGGCACAGTCAAGG-3' (forward) \\
& 5'-CTCAGCACCAGCATCACC-3' (reverse)
\end{tabular}

${ }^{\text {a} H u m a n ~ b o n e ~ m o r p h o g e n e t i c ~ p r o t e i n ~} 2$ (hBMP2) gene and glyceraldehyde-3-phosphate dehydrogenase (GAPDH) were designed as the primer set for qPCR analysis to detect the relative mRNA expression.

osteoid granules. The density of hBMP2 proteins secreted by the rBMSCs with OI media was detected in each group ( $\mathrm{n}=5$ per group) on the 7th and 9th day of OI, followed by the transplantation of the cells on the 9th day. The groups included P3' rBMSCs in the OE group, P3' rBMSCs in the NC group and P3 rBMSCs in MSCs OVX. The rats hBMP2 ELISA kit (R\&D Systems, Minneapolis, MN, USA) was used to detect the density of hBMP2 proteins. The media were changed every $24 \mathrm{~h}$ during the ELISA detection period.

rBMSCs transplantation. The ovaries of $35 \mathrm{SD}$ rats were removed six months later and were denoted second MSCs OVX. After three months, seven rats were randomly selected from the second MSCs OVX to be compared with the second MSCs $\mathrm{CON}(\mathrm{n}=7)$ and second SHAM CON $(\mathrm{n}=7)$ via the experimental indices described above. After construction of the osteoporosis models, the remaining rats in the second MSCs OVX group $(n=28)$ were equally divided into four groups. These groups ( $n=7$ per group) were designated as: transplanted group of $\mathrm{OE}$ cells (group of genetic infection in vitro), transplanted group of $\mathrm{NC}$ cells, injected group of Lv-BMP (group of genetic infection in vivo) and transplanted group of MSCs OVX cells.

The rats were anesthetized with $5 \%$ chloral hydrate in $0.66 \mathrm{ml} / 100 \mathrm{~g}$ through introperitoneal injection. The rats were placed in prone position and the posterolateral side of the hip was chosen for the surgical approach. After the skin, fascia and muscular tissues were removed, we found the trochanter and smoothed its interior. The interior part of the trochanter connected with the femoral neck was determined to be the point of penetration. A mini electric drill (E-504, Shuangxi,
Shanghai, China) containing a 1-mm diameter drill was used to rub the cortical bone in order to expose the spongy bone at this point. A syringe needle was then used to drill through this point.

To achieve an improved effect in treating local osteoporosis, without introducing side effects such as cyst-like bone formation and soft tissue swelling, the density of hBMP2 proteins in the marrow cavity had to be maintained at $\sim 0.15 \mathrm{mg} / \mathrm{ml}(13,14)$. Therefore, the total number of hBMP2 proteins secreted by the transplanted cells had to be maintained at $\sim 0.12 \mathrm{mg}$ in the adult rat femoral cavity. The number of transplanted rBMSCs $\left(5.5 \times 10^{8}\right.$ cells) in the femur was counted, according to the average $\mathrm{hBMP} 2$ protein expression level of the OE group, which was counted via ELISA analysis on the 9th day of the OI. On the 9th day of OI, suspensions of P3 (P3') rBMSCs from three groups (OE, NC and MSCs OVX) were separately created with $0.5 \mathrm{ml}$ saline at a density of $1.1 \times 10^{9} / \mathrm{ml}$. The $10 \mu \mathrm{l}$ finnpipettes (MC, Anting finnpipettes factory, Shanghai, China) were used to inject the rBMSCs suspensions in the left femurs of three corresponding groups (group of genetic infection in vitro, transplanted group of NC cells and transplanted group of MSCs OVX cells). Furthermore, phosphate-buffered saline was injected in the right femurs of all four groups. The range of the injection (from the spongy bone on the top of femur to the spongy bone in the bottom) was divided into five equal sections, where $0.1 \mathrm{ml}$ of the suspension was injected in each section. Some scraps were made by muscle and fascia tissues, which were used to fill the point left by the drill. After the incisions were washed by saline, they were sutured.

The amount of Lv-BMP liquid $(8 \mu \mathrm{l})$ injected in the femur in the group of genetic infection in vivo was calculated according to the average amount of P0 rBMSCs in the femur of MSCs OVX, which was calculated by CFU-Fs counting. The $10 \mu \mathrm{l}$ finnpipettes were used to inject $1.6 \mu \mathrm{l}$ of the Lv-BMP liquid in each section according to the method described above. After the rats were awake, they were fed with fresh water and kept in separate cages. A total of 160,000 units of penicillin was injected in each rat's inner thigh via intramuscular injection (two times a day, for 7 days) and they were then left in their groups. After $\sim 2$ weeks, the walking abilities of all the rats were recovered.

Whole femoral BMD. After three months, the rats were anesthetized by $5 \%$ chloral hydrate in $0.66 \mathrm{ml} / 100 \mathrm{~g}$ through introperitoneal injection. The femoral BMD was measured 
Table III. Statistical results of experiment indices after the first construction of the postmenopausal osteoporosis models.

\begin{tabular}{lccr}
\hline Experiment indices $(\mathrm{n}=7)$ & MSCs CON & MSCs OVX & SHAM CON \\
\hline Content of estrogens (OD) & $0.670 \pm 0.01$ & $0.563 \pm 0.047^{\mathrm{a}}$ & $0.653 \pm 0.023^{\mathrm{c}}$ \\
Wet weight of uterus (g) & $0.342 \pm 0.025$ & $0.133 \pm 0.007^{\mathrm{a}}$ & $0.336 \pm 0.057^{\mathrm{c}}$ \\
Dry weight of femur $\left(\mathrm{g} / \mathrm{cm}^{3}\right)$ & $1.58 \pm 0.08$ & $1.24 \pm 0.03^{\mathrm{b}}$ & $1.55 \pm 0.11^{\mathrm{c}}$ \\
BMD $\left(\mathrm{g} / \mathrm{cm}^{2}\right)$ & $0.236 \pm 0.008$ & $0.178 \pm 0.004^{\mathrm{a}}$ & $0.227 \pm 0.006^{\mathrm{c}}$
\end{tabular}

${ }^{\mathrm{a}} \mathrm{P}<0.05$ as compared with normal (MSCs CON) and osteoporotic (SHAM CON) groups. ${ }^{\text {b }}<0.01$ as compared with MSCCON and SHAM CON . ${ }^{\mathrm{c}} \mathrm{P}<0.05$ as compared with MSCs $\mathrm{CON}$.

Table IV. Statistical results of experiment indices after the second construction of the postmenopausal osteoporosis models.

\begin{tabular}{lccc}
\hline Experiment indices $(\mathrm{n}=7)$ & 2nd MSCs CON & 2nd MSCs OVX & 2ndSHAM CON \\
\hline Content of estrogens (OD) & $0.639 \pm 0.041$ & $0.522 \pm 0.031^{\mathrm{a}}$ & $0.627 \pm 0.063^{\mathrm{c}}$ \\
Wet weight of uterus (g) & $0.331 \pm 0.018$ & $0.205 \pm 0.027^{\mathrm{a}}$ & $0.329 \pm 0.015^{\mathrm{c}}$ \\
Dry weight of femur $\left(\mathrm{g} / \mathrm{cm}^{3}\right)$ & $1.53 \pm 0.12$ & $1.21 \pm 0.007^{\mathrm{b}}$ & $1.49 \pm 0.16^{\mathrm{c}}$ \\
BMD $\left(\mathrm{g} / \mathrm{cm}^{2}\right)$ & $0.243 \pm 0.015$ & $0.171 \pm 0.005^{\mathrm{a}}$ & $0.24 \pm 0.011^{\mathrm{c}}$ \\
\hline
\end{tabular}

${ }^{\mathrm{a}} \mathrm{P}<0.05$ as compared with 2nd normal (MSCs CON) and 2nd osteoporotic (SHAM CON) groups; ${ }^{\mathrm{b}} \mathrm{P}<0.01$ as compared with $2 \mathrm{nd}$ MSCs CON and 2nd SHAM CON; ${ }^{\mathrm{P}}>0.05$ as compared with 2 nd MSCs CON.

in the rats through a dual energy X-ray absorptiometry (QDR-2000 PLUS, Hologic Inc., Waltham, MA, USA) with the rat whole body software package (version 5.73).

Bone histomorphometry analysis. Bone histomorphometry was used in the distal part of the femur $(28,29)$ to compare the change of trabeculars in the groups. The femur was fixed in $70 \%$ ethanol and was cut in half through the median sagittal plane. The experimental material, which was located 3-7 mm from the lowest point of the growth plate and $1 \mathrm{~mm}$ from the lateral cortex, was collected, with the exception of the endocortical region. These materials were embedded and were classified as undecalcified bone sections. A microtome (SM 2000R, Leica, Mannheim, Germany) was used to prepare the bone sections. A total of 12 sections $(5 \mu \mathrm{m})$, were prepared from each material. Two sections were randomly selected and placed on a slide and a total of six slides were prepared. The first and the second sections were used to prepare the toluidine blue stain. The third and fourth sections were used to prepare the hematoxylin and eosin stain (HE stain). The 5th and 6th sections were used to prepare the bone histomorphometry. Five visual fields (the selected area was the secondary spongiosa area, which was rich in trabecular bone) were randomly selected under microscope for each slide and a digital camera connected to the microscope was used to capture images. A computerized automatic image analyzing system (VIDAS2.1, Opton, Germany) was used to detect the area percentage of the bone trabeculars (Tb.Ar/T. Ar), trabecular number (Tb.N), trabecular thickness (Tb.Th), and trabecular separation (Tb.Sp).

Statistical analysis. Data are expressed as means \pm standard deviation (SD). Statistical analysis was performed by analysis of variance (ANOVA) using SPSS16.0 statistical software. Differences were considered statistically significant when $\mathrm{P}<0.05$.

\section{Results}

Osteoporosis models. The PO models of SD rats were constructed twice. The first construction of PO models (Table III) was performed before the isolation of rBMSCs, and the second construction of PO models (Table IV) was performed before the rBMSCs transplantation. There were no statistical differences between MSCs CON (2nd MSCs $\mathrm{CON}$ ) and SHAM CON (2nd SHAM CON) (P>0.05), thus the influence of the surgery on the result of PO models was eliminated. The results showed that the serum content of estrogens and uterus wet weight were lower in MSCs OVX (2nd MSCs OVX) as compared to that of the MSCs CON (2nd MSCs $\mathrm{CON})(\mathrm{P}<0.05)$, suggesting that the content of estrogens clearly decreased after the construction of PO models. Furthermore, the observation of a lower BMD in the right femoral distal 1/3 section and left femur dry weight in MSCs OVX (2nd MSCs OVX) as compared to that of the MSCs CON (2nd MSCs $\mathrm{CON})(\mathrm{P}<0.05)$ suggested a decrease of bone tissue quantity in unit volume after the construction of PO models. The results of these experimental indices (Tables III and IV; Fig. 2) demonstrated that the construction of PO models was successful.

Counting of CFU-Fs, osteoblast ALP staining and osteogenic capability analysis. One CFU-F is formed by cells proliferating from a single $\mathrm{P} 0$ of BMSCs in vitro, such that the number of CFU-Fs stands for the number of BMSCs in marrow cavity $(30,31)$. The number of CFU-Fs in MSCs CON and MSCs 

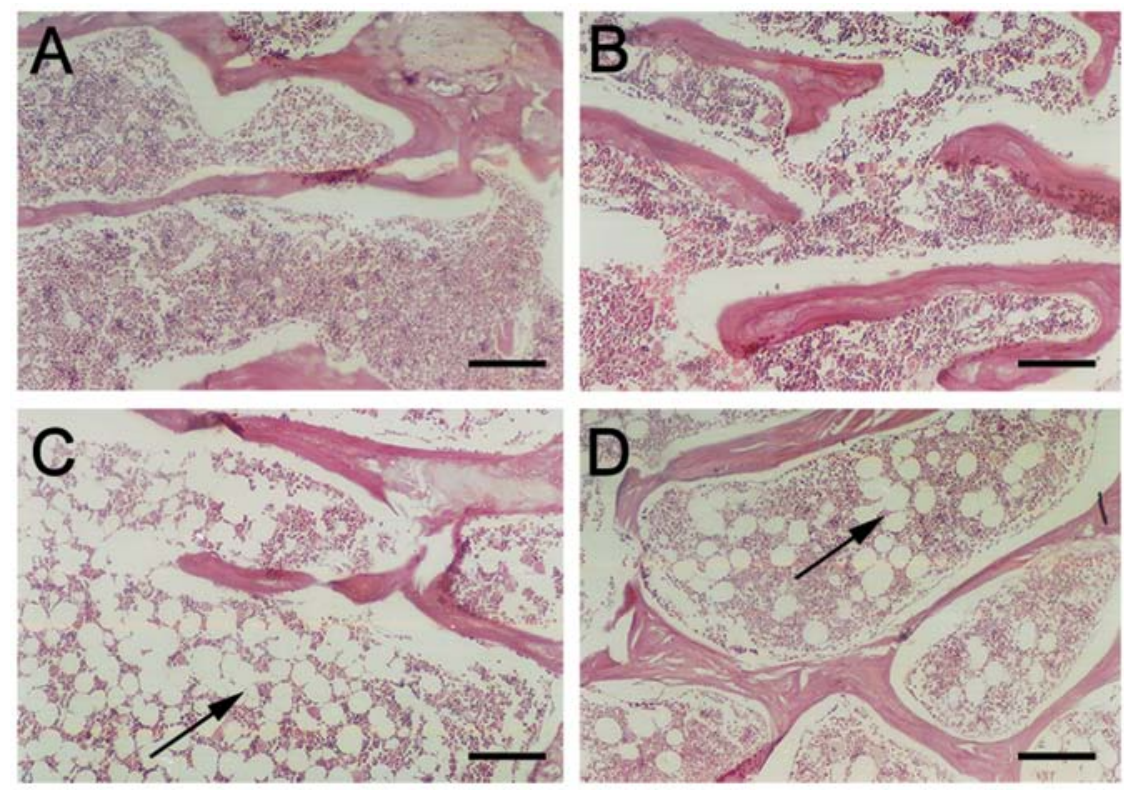

Figure 2. The results of hematoxylin and eosin (H\&E) staining for right femur in (A) normal (MSCs CON), (B) sham (SHAM CON), (C) osteoporosis (MSCs OVX) groups and (D) osteoporosis group after the second construction of postmenopausal osteoporosis models (2nd MSCs OVX). Bone trabeculars of MSCs OVX (2nd MSCs OVX) exhibited symptoms of osteoporosis including thinning, rupture, sparsity and blind ends (Bar, $250 \mu \mathrm{m}$ ).

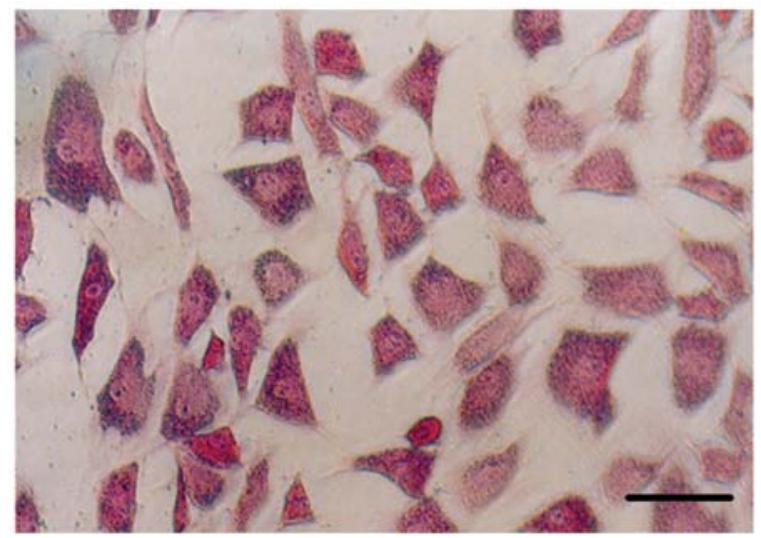

Figure 3. The results of alkaline phosphatase (ALP) staining for rat bone marrow stromal cells (rBMSCs) after osteogenic induction (OI). Over $90 \%$ of the cells in each group presented as ALP positive and there were dark gray or dark black granules in the cytoplasm of osteoblasts in each group. (Bar, $25 \mu \mathrm{m}$ ).

OVX were 53.74 $\pm 3.95 /$ well and 39.89 $\pm 1.72 /$ well, respectively. The average amount of rBMSCs in the MSCs OVX and MSCs CON femurs $\left(1.6 \times 10^{4} \pm 153.27\right.$ in MSCs OVX vs. $2.1 \times 10^{4} \pm 114.6$ in MSCs CON, $\mathrm{P}<0.01)$ was counted according to the number of CFU-Fs.

The OI osteoblasts in each group presented stacked layouts and there were no differences in the shape of the cells or in their CFU-F growth form between the two groups. Over $90 \%$ of the cells in each group presented as ALP-positive stained and there were dark gray or dark black granules in the cytoplasm of the osteoblast in each group (Fig. 3). There was no difference between the two groups after OI and ALP staining.

An important osteogenic indication of BMSCs may be that the cells synthesized and secreted special osteogenic proteins and extracellular matrices including ALP, type I collagen, BMP (appeared early in the process of bone formation)

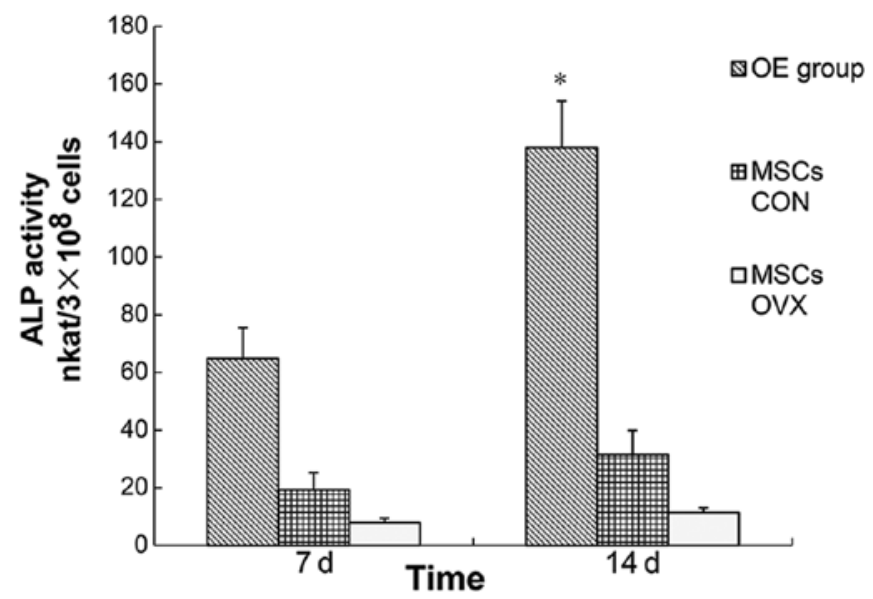

Figure 4. Detection of alkaline phosphatase (ALP) activity for each group [overexpressed (OE), normal (MSCs CON) and osteoporosis (MSCs OVX) groups] on the seventh and fourteenth day of osteogenic induction (OI). On the seventh day of OI, the P-values for different comparisons were: MSCs CON vs. MSCs OVX $(\mathrm{P}<0.05)$, OE group vs. MSCs CON $(\mathrm{P}<0.05)$. On the 14th day of OI, the P-values for different comparisons were: MSCs CON vs. MSCs OVX $(\mathrm{P}<0.05),{ }^{*} \mathrm{OE}$ vs. MSCs CON $(\mathrm{P}<0.01)$. Results are shown as means $\pm \mathrm{SD}$ ( $\mathrm{n}=5$ for each group).

and OCN (appeared late). ALP has been reported to reflect the osteogenic level of BMSCs $(32,33)$ and OCN has been reported to promote a normal process of mineralization in bone (34). We detected ALP activity (Fig. 4) and the OCN level (Fig. 5) to reflect the osteogenic ability of rBMSCs. When the osteogenic capabilities were detected in MSCs CON and MSCs OVX, statistical differences were evident (ALP activity: 29.14 vs. $10.15 \mathrm{nkat} / 3 \times 10^{8}$ cells, $\mathrm{P}<0.05$; OCN level: 8.35 vs. $2.47 \mathrm{ng} / 3 \times 10^{5}$ cells, $\left.\mathrm{P}<0.05\right)$. Therefore, if rBMSCs in MSCs OVX were used to treat local osteoporosis, the effect would have not been ideal. Since BMP gene delivery increased ALP 


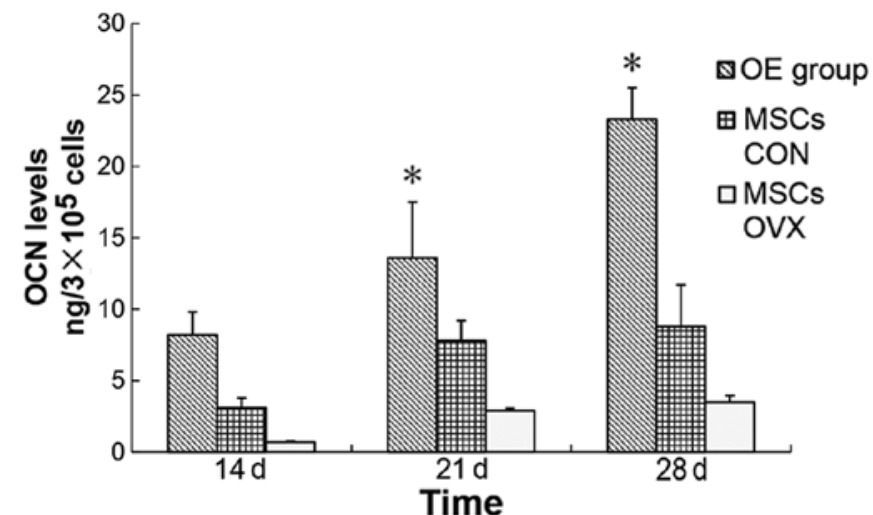

Figure 5. Detection of osteocalcin (OCN) levels for each group [overexpressed (OE), normal (MSCs CON) and osteoporosis (MSCs OVX) groups] on the 14th, 21st, and 28th day of osteogenic induction (OI). On the 14th day of OI, the P-values for different comparisons were: MSCs CON vs. MSCs OVX $(\mathrm{P}>0.05)$, OE group vs. MSCs CON $(\mathrm{P}<0.05)$. On the 21 st day of OI, the P-values for different comparisons were: MSCs CON vs. MSCs OVX $(\mathrm{P}<0.05),{ }^{*} \mathrm{OE}$ group vs. MSCs CON $(\mathrm{P}<0.01)$. On the 28th day of OI, the P-values for different comparisons were: MSCs CON vs. MSCs OVX $(\mathrm{P}<0.05),{ }^{*} \mathrm{OE}$ group vs. MSCs CON $(\mathrm{P}<0.01)$. Results are shown as means $\pm \mathrm{SD}$ ( $\mathrm{n}=5$ for each group).

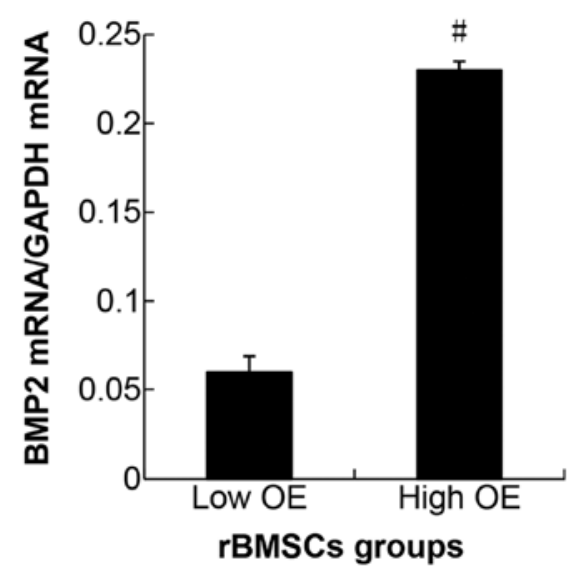

Figure 6. Relative mRNA expression of human bone morphogenetic protein 2 (hBMP2) in rat bone marrow stromal cells (rBMSCs) for each group (low $\mathrm{OE}$, high $\mathrm{OE}$ ) at seven days after the infection. The results are expressed as a ratio to the mRNA levels of the reference gene, GAPDH. For the high vs. low $\mathrm{OE}$ group, $\mathrm{P}<0.05$. Data are expressed as means $\pm \mathrm{SD}$ ( $\mathrm{n}=3$ for each group).

expression in BMSCs (35), we constructed Lv-BMP to infect the rBMSCs in MSCs OVX and the osteogenic capabilities between the OE group and MSCs CON were compared. The results of this comparison indicated that the osteogenic capability of rBMSCs in the OE group markedly increased and statistical differences (ALP activity: 137.57 vs. $29.14 \mathrm{nkat} / 3 \times 10^{8}$ cells, $\mathrm{P}<0.01$; OCN level: 24.16 vs. $8.35 \mathrm{ng} / 3 \times 10^{5}$ cells, $\left.\mathrm{P}<0.01\right)$ were reported as compared to that of the MSCs CON. Therefore, if rBMSCs were used as the transplanted materials in order to treat the local osteoporosis, rBMSCs in the OE group would potentially be more appropriate.

$q P C R$ and ELISA. The qPCR results are shown in Fig. 6. The mRNA expression level of hBMP2 gene in the control and NC groups was undetectable. A significant difference of hBMP2 expression was detected between the high and low

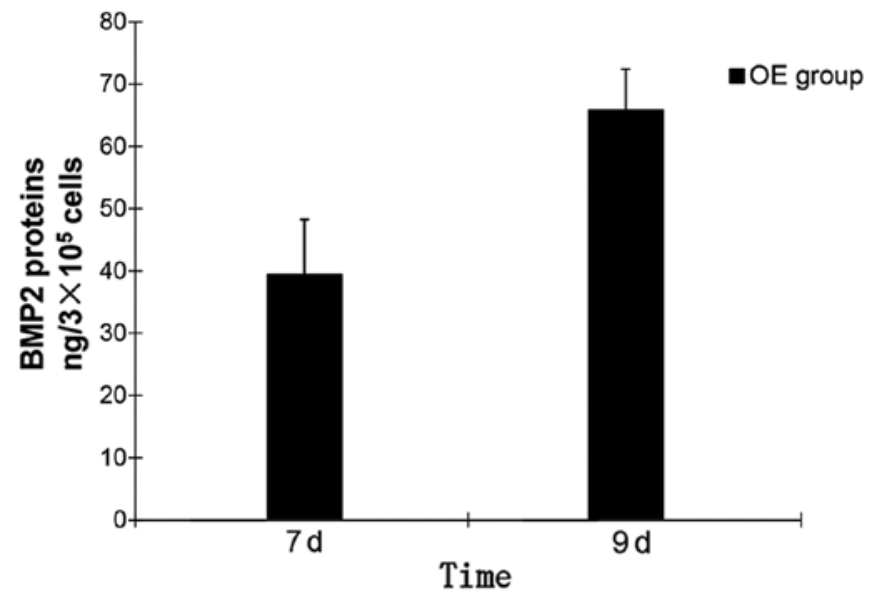

Figure 7. Supernatants of rat bone marrow stromal cells (rBMSCs) in overexpressed (OE) group were collected on the 7th and 9th day of osteogenic induction (OI) and measured with ELISA kits specific for bone morphogenetic protein 2 (BMP). OE group, hBMP2 overexpressed group. Results are shown as means $\pm \mathrm{SD}$ ( $\mathrm{n}=5$ for each group)

OE groups $(\mathrm{P}<0.05)$. As a result, the mRNA expression level of the hBMP2 gene reflected a higher lentiviral gene transfer efficiency that was obtained at a MOI of 40. On the 9th day of OI, the ELISA result (Fig. 7) showed that the density of $\mathrm{hBMP} 2$ proteins in the OE group was $65.79 \mathrm{ng} / 3 \times 10^{5}$ cells on average. This indicated that $1 \times 10^{5}$ cells in OE group secreted an average of $21.93 \mathrm{ng}$ hBMP2 protein on the 9th day of OI. The density of these hBMP2 proteins in MSCs OVX and the NC group were negative. The qPCR and ELISA results showed that the hBMP2 gene was successfully expressed in rBMSCs through lentivirus-mediated infection.

Whole femoral BMD. Results of the whole femoral BMD (Fig. 8) suggested that the group of genetic infection in vitro had a higher expression as compared to the group of genetic infection in vivo $(\mathrm{P}<0.01)$. There were no statistical differences $(\mathrm{P}>0.05)$ between the transplanted group of MSCs OVX cells and transplanted group of NC cells, thus they were designated as the transplanted group of OVX cells (NC cells). The whole femoral BMD in the transplanted group of OVX cells (NC cells) was higher than that of the group of genetic infection in vivo $(\mathrm{P}<0.05)$. Statistical differences $(\mathrm{P}<0.05)$ were observed between the group of genetic infection in vivo and the control group of saline.

Bone histomorphometry. The results of bone histomorphometry (Fig. 9) suggested there were no statistical differences in Tb.N ( $P>0.05)$ between the groups. Results of the other three structural bone histomorphometric parameters (Tb.Ar/T. Ar, Tb.Th, Tb.Sp) showed that the group of genetic infection in vitro had the highest Tb.Ar/T.Ar and Tb.Th among all the groups, but the lowest Tb.Sp. Obvious statistical differences $(\mathrm{P}<0.01)$ were observed between the group of genetic infection in vitro and the remaining groups. No statistical differences ( $P>0.05)$ were observed between the transplanted group of MSCs OVX cells and the transplanted group of NC cells, thus these two groups were designated as the transplanted group of OVX cells (NC cells). The transplanted group of OVX 


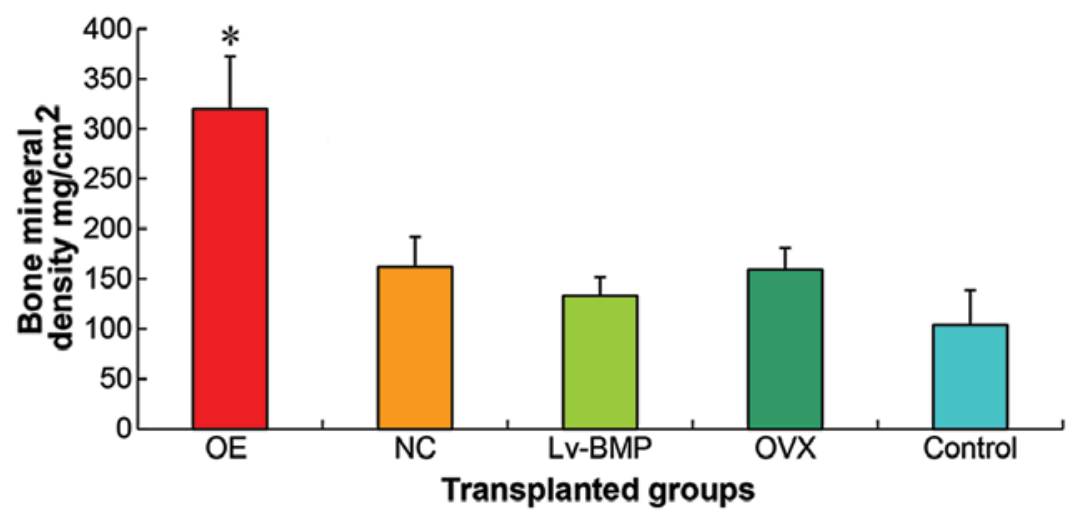

Figure 8. Whole femoral bone mineral density (BMD) results for each group after rat bone marrow stromal cells (rBMSCs) transplantation. OE, group of genetic infection in vitro; NC, transplanted group of NC cells; lentivirus containing hBMP2 cDNA (Lv-BMP), group of genetic infection in vivo; OVX, transplanted group of MSCs OVX cells; CON, control group of saline. "For OE vs. Lv-BMP, $\mathrm{P}<0.01$. Results are expressed as means $\pm \mathrm{SD}$ ( $\mathrm{n}=7$ for each group).
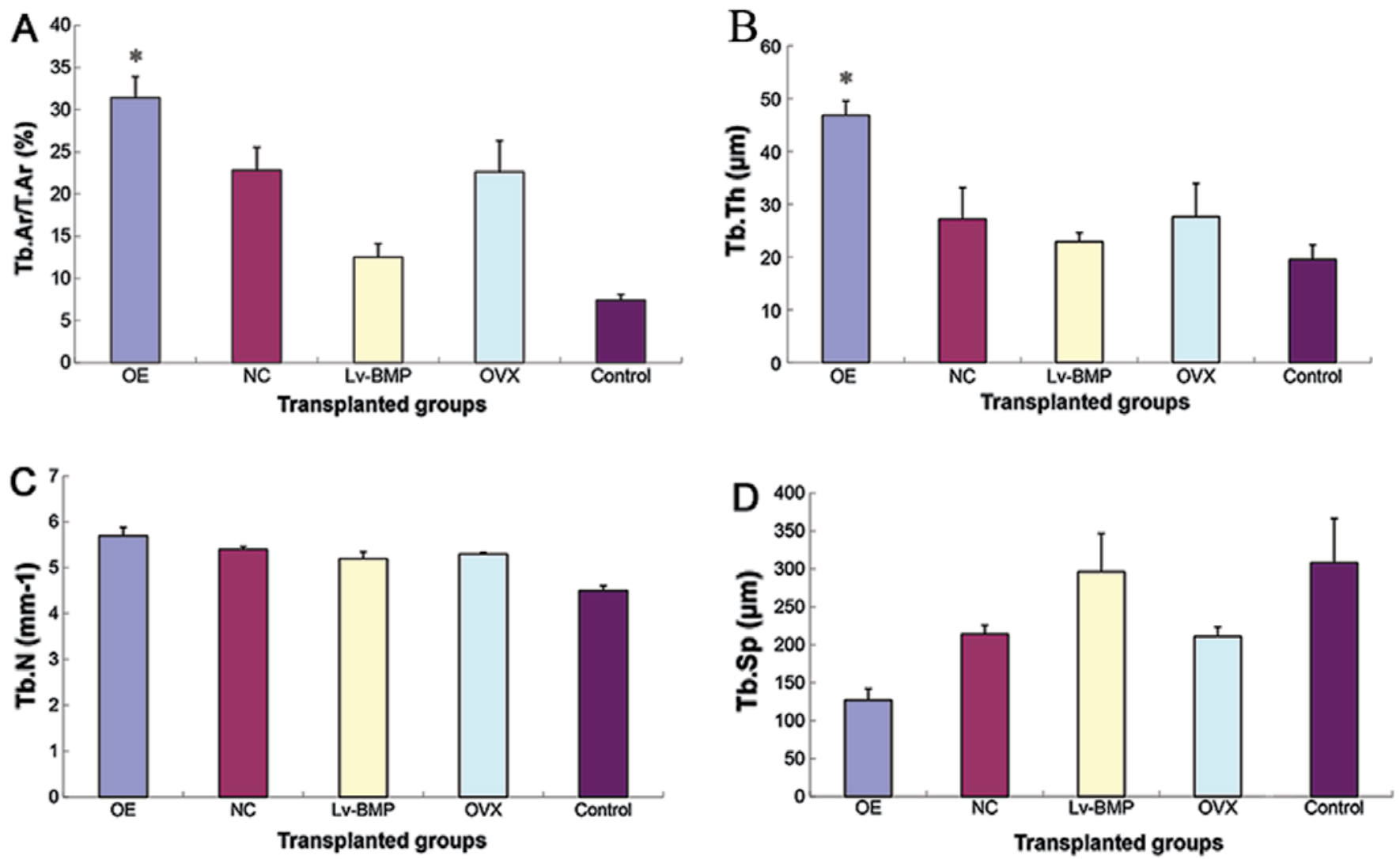

Figure 9. Bone histomorphometry results for each group after rBMSCs transplantation. Overexpressed (OE), group of genetic infection in vitro; NC, transplanted group of NC cells; lentivirus containing hBMP2 cDNA (Lv-BMP), group of genetic infection in vivo; OVX, transplanted group of MSCs OVX cells; CON, control group of saline. (A) The area of trabecular bones in percentage (Tb.Ar/T.Ar), (B) trabecular thickness (Tb.Th), (C) trabecular number (Tb.N) and (D) trabecular separation (Tb.Sp). "For OE vs. Lv-BMP, $\mathrm{P}<0.01$. Results are expressed as means $\pm \mathrm{SD}$ ( $\mathrm{n}=7$ for each group).

cells (NC cells) had obvious statistical differences $(\mathrm{P}<0.01)$ with respect to $\mathrm{Tb}$.Ar/T.Ar, Tb.Sp as compared to the group of genetic infection in vivo and the control group of saline. Similarly, statistical difference $(\mathrm{P}<0.05)$ with respect to $\mathrm{Tb} . \mathrm{Th}$ was observed. The group of genetic infection in vivo had statistical differences $(\mathrm{P}<0.05)$ with respect to the $\mathrm{Tb}$.Ar/T. $\mathrm{Ar}$ and $\mathrm{Tb} . \mathrm{Th}$ as compared to the control group of saline. However, with respect to the $\mathrm{Tb} . \mathrm{Sp}$, no statistical differences were observed $(\mathrm{P}>0.05)$.
Femoral section stain. The femoral sections were stained by toluidine blue and HE stains (Fig. 10). The bone trabeculars in the group of genetic infection in vitro were thicker, closer and in alignment as compared to the other groups, while the symptoms of osteoporosis, such as rupture and blind ends occured less than that of the other groups. The bone trabeculars in the group of genetic infection in vivo were the most similar to those of the control group of saline. The shape of the bone trabeculars exhibited symptoms of osteoporosis such 

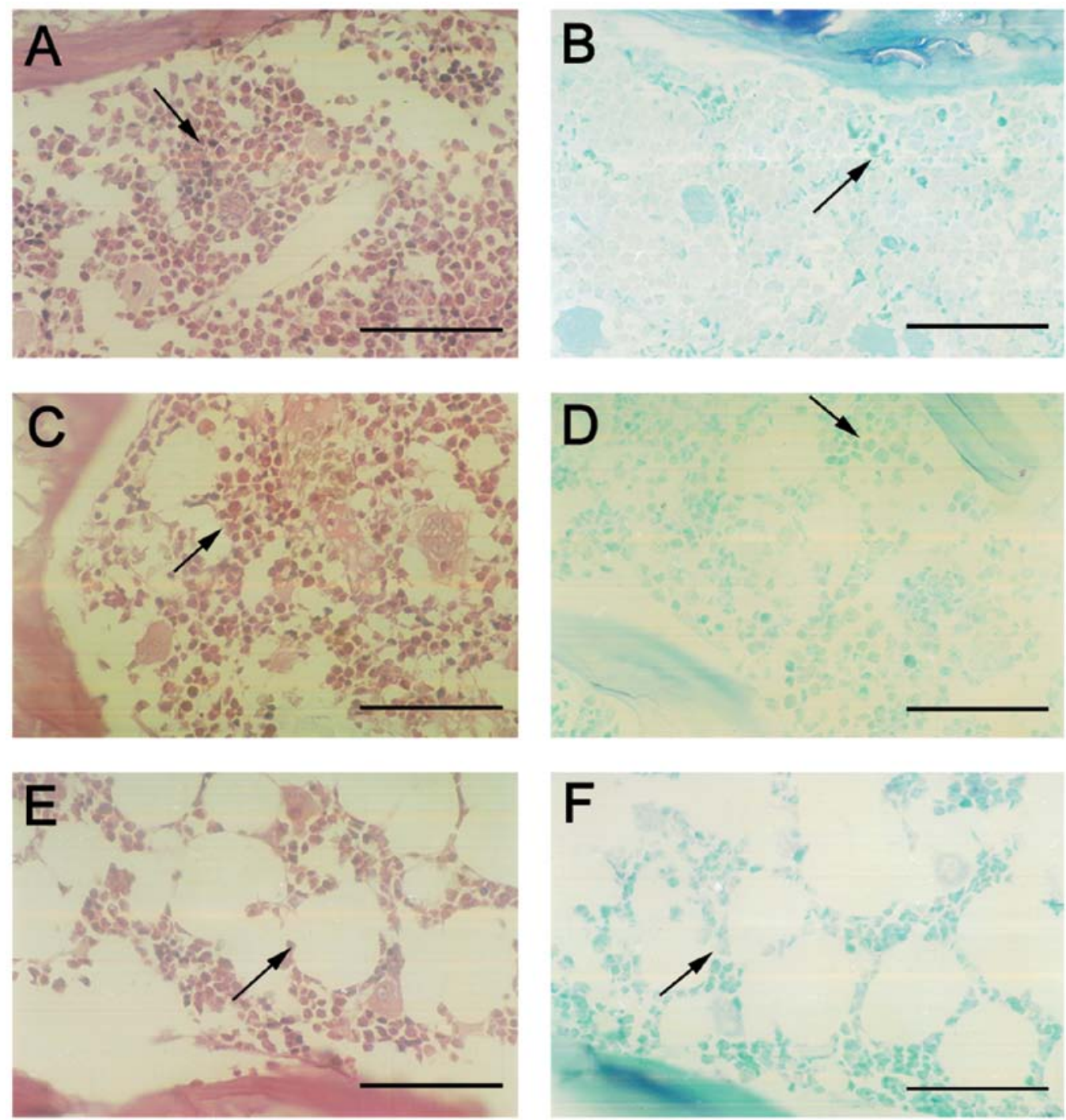

Figure 10. Hematoxylin and eosin (H\&E) and toluidine blue stain as a result of rat femoral section staining after rat bone marrow stromal cell (rBMSCs) transplantation (Bar, $50 \mu \mathrm{m}$ ). Group of genetic infection (A and B) in vitro and (C and D) in vivo. (E and $\mathrm{F}$ ) Control group of saline.

as thinning, rupture, sparsity and blind ends. No cyst-like bone formation and soft tissue swelling were observed.

\section{Discussion}

The present study demonstrated that the treatment effect of Lv-BMP-mediated hBMP2 gene overexpression in the osteoporotic rBMSCs, which was created by in vitro genetic infection in local osteoporosis was improved compared with that created by genetic infection in vivo. We analyzed three possible causes for this observation. The amount of rBMSCs in MSCs OVX and MSCs CON (1.6x10 \pm 153.27 vs. $2.1 \times 10^{4} \pm 114.6$, $\mathrm{P}<0.01)$ suggested that $\mathrm{rBMSCs}$ in the femoral marrow cavity of MSCs OVX were rare. Even if all the rBMSCs were to be infected, the hBMP2 proteins secreted by these cells could not have achieved the level $[0.15 \mathrm{mg} / \mathrm{ml}(13,14)]$ required for the treatment of local osteoporosis. However, if the rBMSCs were isolated from the MSCs OVX, and infected by Lv-BMP in vitro, the infected rBMSCs would have increased and the hBMP2 proteins secreted by them would have achieved the level needed for the treatment of the local osteoporosis. The
OI of rBMSCs may therefore have been conducted in vitro. A comparison of the rBMSCs in MSCs OVX to MSCs CON in terms of osteogenic capability, would yield a lower ability in the rBMSCs in MSCs OVX compared with those in MSCs CON $(\mathrm{P}<0.01)$, whereas a comparison of the rBMSCs with OI in these two groups, may yield a statistical difference $(\mathrm{P}<0.05)$, that may have been not so obvious (36). This result may have been a possible indication that the osteogenic ability of the rBMSCs with OI in MSCs OVX approached that of the MSCs CON. The Lv-BMP infection efficiency was also mentioned. The rBMSC quantity was different in the MSCs OVX of individual rat femurs. Additionally, the individualized quantity of Lv-BMP could not be evaluated. Subsequently, the Lv-BMP infection efficiency was not proven. Even if the individualized quantity of Lv-BMP had been realized, Lv-BMP targeting would have to be resolved. The in vivo genetic infection omitted the processes of subculture, OI and genetic infection in vitro. Furthermore, it was convenient and cost-effective; however, the result was not satisfactory. By contrast, the results of the in vitro genetic infection demonstrated an improved treatment effect on local osteoporosis compared with that of the other 
methods employed. This laid a foundation for treating the local osteoporosis of patients by using their own BMSCs.

BMP2 participates in a number of molecular pathways that might contribute to certain adverse clinical effects, including cyst-like bone formation, significant soft tissue swelling, as well as the resorption of excessive bone and vertebral subsidence or collapse $(37,38)$. Since BMP2 is a known chemoattractant for monocytes, macrophages and lymphocytes, its inflammatory response is not unexpected. Furthermore, by potentiating the receptor activation of nuclear factor- $\kappa \mathrm{B}$ ligand (a cytokine essential for inducing osteoclast differentiation), BMP2 is capable of inducing osteoclastogenesis (39). BMP2 is also able to inhibit Wnt signaling by upregulating PPAR $\gamma$, thereby increasing osteoclasts since PPAR $\gamma$ upregulates the expression of c-fos (an essential mediator of osteoclastogenesis) (40). High-dose BMP2 ( $\geq 1.5 \mathrm{mg} / \mathrm{ml}$ ) decreases the osteoblast expression of osteoprotegerin (a member of the tumor necrosis factor receptor family that inhibits the receptor activator of nuclear factor- $\kappa \mathrm{B}$ ligand) by inhibiting Wnt signaling (41). Therefore, high-dose BMP2 induces significant tissue inflammation, which explains the adverse clinical effects including cyst-like bone formation and significant soft tissue swelling, and increases osteoclastogenesis, which may clinically manifest as vertebral subsidence, collapse and excessive bone resorption.

In the present study, hBMP2 proteins at a density of $0.15 \mathrm{mg} / \mathrm{ml}$ were enough to produce osteogenesis by potentiating the rBMSCs transplanted or contained through autocrine or paracrine effects. At this density, hBMP2 had treatment effects on the local femoral osteoporosis, with the exception of adverse clinical effects including cyst-like bone formation and significant soft tissue swelling. However, in order to produce the treatment effect on local osteoporosis in human, the density of hBMP2 proteins was required to be at least $1.5 \mathrm{mg} / \mathrm{ml}$ in the bone marrow cavity. Thus, clinical adverse effects could be introduced at this high dose of hBMP2 proteins. Furthermore, the quality of regenerated bone could be reduced, with the cyst-like bone void formation and inflammation resulting in life-threatening outcomes. Therefore, the method of in vitro genetic infection with human BMSCs through the lentivirus-carrying hBMP2 gene in order to treat human local osteoporosis is not appropriate. However, if this in vitro method of genetic infection were used to infect human BMSCs with the hBMP2 gene and other growth factors in order to suppress the non-osteogenic BMP2 signaling targets or to decrease the BMP2 dose requirement, unwanted inflammatory and adipogenetic induction could be reduced. Using this approach, improvements in the treatment of human local osteogenesis could be achieved.

In conclusion, the results of this study have shown that the hBMP2-modified osteoporotic rat BMSCs formed by in vitro genetic infection had an improved treatment effect as compared with that of the in vivo genetic infection (BMD: 0.315 vs. $0.19 \mathrm{~g} / \mathrm{cm}^{2}, \mathrm{P}<0.01$; bone histomorphometry: Tb.Ar/T. Ar: 0.301 vs. $0.114, \mathrm{P}<0.01$. Tb.Th: 43.54 vs. $21.39 \mu \mathrm{m}, \mathrm{P}<0.01$. Tb.Sp: 115.7 vs. $304.87 \mu \mathrm{m}, \mathrm{P}<0.01)$. We concluded that the treatment effect of the Lv-BMP-mediated hBMP2 gene that was overexpressed in osteoporotic rBMSCs formed by in vitro genetic infection on local osteoporosis was better than that performed by in vivo genetic infection.

\section{Acknowledgements}

This study was supported by the National Science Foundation of China (grant no. NFSC-30227738) and a grant from the Heilongjiang Education Bureau, China (grant no. 12521180). The authors would like to thank Professor Tianzun Tao and Wanli Tian for assistance in collecting the experimental data.

\section{References}

1. Kim S, Kang Y, Krueger CA, et al: Sequential delivery of BMP-2 and IGF-1 using a chitosan gel with gelatin microspheres enhances early osteoblastic differentiation. Acta Biomater 8: 1768-1777, 2012.

2. Song I, Kim BS, Kim CS and Im GI: Effects of BMP-2 and vitamin $\mathrm{D} 3$ on the osteogenic differentiation of adipose stem cells. Biochem Biophys Res Commun 408: 126-131, 2011.

3. Edgar CM, Chakravarthy V, Barnes G, Kakar S, Gerstenfeld LC and Einhorn TA: Autogenous regulation of a network of bone morphogenetic proteins (BMPs) mediates the osteogenic differentiation in murine marrow stromal cells. Bone 40: 1389-1398, 2007.

4. Ben-David D, Srouji S, Shapira-Schweitzer K, et al: Low dose BMP-2 treatment for bone repair using a PEGylated fibrinogen hydrogel matrix. Biomaterials 34: 2902-2910, 2013.

5. Tsuji K, Bandyopadhyay A, Harfe BD, et al: BMP2 activity, although dispensable for bone formation, is required for the initiation of fracture healing. Nat Genet 38: 1424-1429, 2006.

6. Matsumoto A, Yamaji K, Kawanami M and Kato H: Effect of aging on bone formation induced by recombinant human bone morphogenetic protein-2 combined with fibrous collagen membranes at subperiosteal sites. J Periodontal Res 36: 175-182, 2001.

7. Bai Y, Li P, Yin G, et al: BMP-2, VEGF and bFGF synergistically promote the osteogenic differentiation of rat bone marrow-derived mesenchymal stem cells. Biotechnol Lett 35: 301-308, 2013.

8. Lieberman JR, Daluiski A, Stevenson S, et al: The effect of regional gene therapy with bone morphogenetic protein-2-producing bone-marrow cells on the repair of segmental femoral defects in rats. J Bone Joint Surg Am 81: 905-917, 1999.

9. Tsuda H, Wada T, Ito Y, et al: Efficient BMP2 gene transfer and bone formation of mesenchymal stem cells by a fiber-mutant adenoviral vector. Mol Ther 7: 354-365, 2003.

10. Jiang J, Fan CY and Zeng BF: Osteogenic differentiation effects on rat bone marrow-derived mesenchymal stromal cells by lentivirus-mediated co-transfection of human BMP2 gene and VEGF165 gene. Biotechnol Lett 30: 197-203, 2008.

11. Zhu C, Chang Q, Zou D, et al: LvBMP-2 gene-modified BMSCs combined with calcium phosphate cement scaffolds for the repair of calvarial defects in rats. J Mater Sci Mater Med 22: 1965-1973, 2011.

12. Hsu WK, Sugiyama O, Park SH, et al: Lentiviral-mediated BMP-2 gene transfer enhances healing of segmental femoral defects in rats. Bone 40: 931-938, 2007.

13. Oluigbo CO and Solanki GA: Use of recombinant human bone morphogenetic protein-2 to enhance posterior cervical spine fusion at 2 years of age: technical note. Pediatr Neurosurg 44: 393-396, 2008.

14. Zara JN, Siu RK, Zhang X, et al: High doses of bone morphogenetic protein 2 induce structurally abnormal bone and inflammation in vivo. Tissue Eng Part A 17: 1389-1399, 2011.

15. Tsuda H, Wada T, Yamashita $T$ and Hamada H: Enhanced osteoinduction by mesenchymal stem cells transfected with a fibermutant adenoviral BMP2 gene. J Gene Med 7: 1322-1334, 2005.

16. Yukawa Y, Lou J, Fukui N and Lenke LG: Optimal treatment timing to attenuate neuronal apoptosis via Bcl-2 gene transfer in vitro and in vivo. J Neurotrauma 19: 1091-1103, 2002.

17. Sapet C, Pellegrino C, Laurent N, Sicard F and Zelphati O: Magnetic nanoparticles enhance adenovirus transduction in vitro and in vivo. Pharm Res 29: 1203-1218, 2012.

18. Zhang L, Liu HJ, Li TJ, et al: Lentiviral vector-mediated siRNA knockdown of SR-PSOX inhibits foam cell formation in vitro. Acta Pharmacol Sin 29: 847-852, 2008.

19. Zhou S, Mizuno S and Glowacki J: Wnt pathway regulation by demineralized bone is approximated by both BMP-2 and TGF- $\beta 1$ signaling. J Orthop Res 31: 554-560, 2013. 
20. Jiang XQ, Chen JG, Gittens S, Chen CJ, Zhang XL and Zhang ZY: The ectopic study of tissue-engineered bone with hBMP-4 gene modified bone marrow stromal cells in rabbits. Chin Med J (Engl) 118: 281-288, 2005.

21. Krebsbach PH, Zhang K, Malik AK and Kurachi K: Bone marrow stromal cells as a genetic platform for systemic delivery of therapeutic proteins in vivo: human factor IX model. J Gene Med 5: 11-17, 2003.

22. Hao L, Wang J,Zou Z, et al: Transplantation of BMSCs expressing $\mathrm{hPDGF}-\mathrm{A} / \mathrm{hBD} 2$ promotes wound healing in rats with combined radiation-wound injury. Gene Ther 16: 34-42, 2009.

23. Huang Q, Liu XZ, Kang CS, Wang GX, Zhong Y and Pu PY: The anti-glioma effect of suicide gene therapy using BMSC expressing HSV/TK combined with overexpression of $\mathrm{Cx} 43$ in glioma cells. Cancer Gene Ther 17: 192-202, 2010.

24. Maniatopoulos $\mathbf{C}$, Sodek $\mathbf{J}$ and Melcher AH: Bone formation in vitro by stromal cells obtained from bone marrow of young adult rats. Cell Tissue Res 254: 317-330, 1988.

25. Zou D, Han W, You S, et al: In vitro study of enhanced osteogenesis induced by HIF-1 $\alpha$-transduced bone marrow stem cells Cell Prolif 44: 234-243, 2011.

26. Nishida S, Endo N, Yamagiwa H, Tanizawa T and Takahashi HE: Number of osteoprogenitor cells in human bone marrow markedly decreases after skeletal maturation. J Bone Miner Metab 17: 171-177, 1999.

27. Liu HC, E LL, Wang DS, et al: Reconstruction of alveolar bone defects using bone morphogenetic protein 2 mediated rabbit dental pulp stem cells seeded on nano-hydroxyapatite/collagen/ poly(L-lactide). Tissue Eng Part A 17: 2417-2433, 2011.

28. Tamminen IS, Isaksson H, Aula AS, Honkanen E, Jurvelin JS and Kröger H: Reproducibility and agreement of micro-CT and histomorphometry in human trabecular bone with different metabolic status. J Bone Miner Metab 29: 442-448, 2011.

29. Hapidin H, Othman F, Soelaiman IN, Shuid AN and Mohamed N: Effects of nicotine administration and nicotine cessation on bone histomorphometry and bone biomarkers in Sprague-Dawley male rats. Calcif Tissue Int 88: 41-47, 2011.

30. Owen M and Friedenstein AJ: Stromal stem cells: marrow-derived osteogenic precursors. Ciba Found Symp 136: 42-60, 1988.
31. Derubeis AR and Cancedda R: Bone marrow stromal cells (BMSCs) in bone engineering: limitations and recent advances. Ann Biomed Eng 32: 160-165, 2004

32. Zou L, Zou X, Chen L, et al: Multilineage differentiation of porcine bone marrow stromal cells associated with specific gene expression pattern. J Orthop Res 26: 56-64, 2008.

33. Stucki U, Schmid J, Hämmerle CF and Lang NP: Temporal and local appearance of alkaline phosphatase activity in early stages of guided bone regeneration. A descriptive histochemical study in humans. Clin Oral Implants Res 12: 121-127, 2001.

34. Luu HH, Song WX, Luo X, et al: Distinct roles of bone morphogenetic proteins in osteogenic differentiation of mesenchymal stem cells. J Orthop Res 25: 665-677, 2007.

35. Ying X, Cheng S, Wang W, et al: Effect of boron on osteogenic differentiation of human bone marrow stromal cells. Biol Trace Elem Res 144: 306-315, 2011.

36. Li DJ, Ge DX, Wu WC, Wu J and Li L: [Osteogenic potential of bone marrow mesenchymal stem cells from ovariectomied osteoporotic rat]. Sichuan Da Xue Xue Bao Yi Xue Ban 36: 318-321, 2005 (In Chinese).

37. Vaidya R, Weir R, Sethi A, Meisterling S, Hakeos W and Wybo CD: Interbody fusion with allograft and rhBMP-2 leads to consistent fusion but early subsidence. J Bone Joint Surg Br 89: 342-345, 2007

38. Vaidya R, Carp J, Sethi A, Bartol S, Craig J and Les CM: Complications of anterior cervical discectomy and fusion using recombinant human bone morphogenetic protein-2. Eur Spine J 16: 1257-1265, 2007.

39. Irie K, Alpaslan C, Takahashi K, et al: Osteoclast differentiation in ectopic bone formation induced by recombinant human bone morphogenetic protein 2 (rhBMP-2). J Bone Miner Metab 21: 363-369, 2003.

40. Wan Y, Chong LW and Evans RM: PPAR-gamma regulates osteoclastogenesis in mice. Nat Med 13: 1496-1503, 2007.

41. Glass DA II and Karsenty G: Canonical Wnt signaling in osteoblasts is required for osteoclast differentiation. Ann N Y Acad Sci 1068: 117-130, 2006. 